\title{
Genetic Mutations in a Patient with Chronic Myeloid Leukemia Showing Blast Crisis 10 Years After Presentation
}

\author{
LISA-MADELEINE SKLARZ, CHRISTOPH WITTKE, SASKIA KROHN, CHRISTINA GROßE-THIE, \\ CHRISTIAN JUNGHANSS, HUGO MURUA ESCOBAR and HARTMUT GLAESER \\ Department of Medicine, Clinic III - Hematology/Oncology/Palliative Medicine, \\ Rostock University Medical Center, Rostock, Germany
}

\begin{abstract}
Since the introduction of tyrosine kinase inhibitors (TKI), the prospects for patients with chronic myeloid leukemia (CML) have improved significantly. Herein we present the case of a patient with CML who experienced blast crisis and development of acute myeloid leukemia (AML) 10 years after presentation. The CML was characterized by the gene fusion of breakpoint cluster region BCR and tyrosineprotein kinase ABL1. During treatment different therapeutic protocols including imatinib, nilotinib, dasatinib and ponatinib were applied due to development of resistance or non-response. Fluorescence in situ hybridization (FISH) and next-generation sequencing (NGS) were used to describe cytogenetic and molecular aberrations elucidating the development into AML: A loss of chromosome 7, as well as an arising frequency of variants in the gene met protooncogene MET (p.T110I) and tyrosine-protein phosphatase non-receptor type 11 PTPN11 (p.Q510L) was observed. This report describes the comprehensive characterization of a clinical case showing multiple therapeutic resistances correlated with genetic aberrations.
\end{abstract}

Most patients with chronic myeloid leukemia (CML) show very good response towards therapy protocols with tyrosine kinase inhibitors (TKI) such as imatinib or nilotinib (1). However, some patients develop resistance to the standard regimen during their clinical course (2). This requires a subsequent change in treatment due to the risk of a blast

Correspondence to: Hugo Murua Escobar/Hartmut Glaeser, Department of Medicine, Clinic III-Hematology/Oncology/Palliative Medicine, Rostock University Medical Center, Ernst-Heydemann-Str. 6, 18057 Rostock, Germany. Tel: +49 3814947421, Fax: +49 3814947422, e-mail: hugo.murua.escobar@med.uni-rostock.de; hartmut.glaeser@med.uni-rostock.de

Key Words: Next-generation sequencing, blast crisis, cytogenetic and molecular aberrations, allogenic stem cell transplantation, clonal development FISH. crisis (3). Furthermore, during the past decade the identification of mutations by next-generation sequencing (NGS) has become a key element in cancer research, allowing sophisticated molecular diagnostics, as well as comprehensive retrospective molecular analysis of interesting cases. Thus, NGS provides the possibility to retrospectively elucidate molecular mechanisms underlying different drug responses, as well as drug resistance mechanisms, clonal development and evolution. Linking NGS data with conventional diagnostic tools such as cytogenetics, fluorescence in situ hybridization (FISH) and their correlation with therapeutic intervention and observed response allows deeper understanding of individual cases.

Here the comprehensive characterization of a clinical case showing multiple types of therapeutic resistance correlated with genetic aberrations is described.

\section{Case Report}

A man (born in 1965) who was diagnosed with CML in May 2004 presented with a blast crisis and acute myeloid leukemia (AML) in February 2015. Conventional diagnostic analyses were performed by cytogenetics and FISH. From March 2015, additional NGS was routinely and retrospectively performed monitoring the initial CML as well as the AML manifestation. Thereby, a comprehensive analysis and evaluation of therapeutic interventions as well as the diagnostic measurements were performed. Over the 10 years analyzed, different therapeutic protocols were used. Therapeutic intervention, NGS analysis and cytogenetic diagnostics are summarized in Figure 1.

$N G S$. DNA for NGS analyses was isolated from nucleus suspensions of bone marrow (Nucleospin Tissue XS; Macherey Nagel, Düren Germany), peripheral blood smears (Nucleospin Tissue, Macherey Nagel) and peripheral blood (NucleoBond CB 100, Macherey Nagel) performed according to the manufacturer's instructions. NGS analysis was performed using Ion AmpliSeq ${ }^{\mathrm{TM}}$ Cancer Hotspot Panel 
v2 (Thermo Fisher Scientific, Schwerte, Germany) and Ion PGM $^{\mathrm{TM}}$ System (Thermo Fisher Scientific). This panel includes 50 genes associated with different cancer types. Furthermore, NGS data were mapped against the human genome (hg19) and analyzed by Ion Reporter ${ }^{\mathrm{TM}}$ Software (Thermo Fisher Scientific, Carlsbad, CA, USA). Only exonic variants with a quality over 60 (Phred-Score) and amplicon coverage above 500 are shown in Figure 1. ClinVar (National Center for Biotechnology Information, National Library of Medicine, Rockville Pike, Bethesda MD, USA) and Functional Analysis Through Hidden Markov Models (FATHMM) prediction were used for data interpretation (4).

Cytogenetics and molecular cytogenetics. Chromosomal analysis on bone marrow was performed using RC-banding (samples until 2009) (5) and GTG-banding (samples after 2009, Department of Human Genetics, University Bremen). A minimum of 20 metaphase cells were analyzed and the karyotype was described according to the 2016 International System for Human Cytogenetic Nomenclature (6).

FISH using the following probes was carried out according to the manufacturers' instructions and 100 interphases (sample August 2014: 300) were routinely counted: Vysis D20S108 (20q12) FISH Probe (Abbott Molecular, Abbott Park, IL, USA); Vysis D7S486/Vysis CEP 7 FISH Probes (Abbott Molecular), Vysis LSI BCR/ABL Dual Color, Dual Fusion Translocation Probe and XCE 8 Blue (MetaSystems, Altlussheim, Germany).

Breakpoint cluster region $(B C R)$ and tyrosine-protein kinase (ABL1) detection. BCR-ABL status was routinely determined according to standard methods (polymerase chain reaction) at the Department of Internal Medicine, Medical Clinic C, Clinic for Hematology, Oncology and Palliative Care, University Medical Center Greifswald. Values are presented in international standard. Bone marrow and peripheral blood were first characterized by cytogenetics and FISH in May 2004.

Clinical course. In May 2004, the patient was found to be $B C R$ $A B L$-positive [BCR-ABL $117 \%$ peripheral blood, karyotype 46XY,t(9;22)(q34;q11)] and was treated with hydroxycarbamide according to the standard protocol. Hydroxycarbamide was replaced with imatinib (200 mg per day) in July 2004. In September 2004, the imatinib dose was increased to $400 \mathrm{mg}$ daily due to persisting $14 \% B C R-A B L$ (peripheral blood). Peripheral blood screening revealed $45 \% B C R-A B L$ in November 2005 and the imatinib dose was further increased to $600 \mathrm{mg}$ daily. Despite dose adaption for a further 2 months (January 2006), a further increase of $B C R-A B L$ to $58 \%$ in bone marrow and $28 \%$ in peripheral blood required a subsequent dose escalation of imatinib to $800 \mathrm{mg}$ per day. Corresponding cytogenetic analyses revealed an additional aberration with a subpopulation of $48, \mathrm{XY},+8, \mathrm{t}(9 ; 22)(\mathrm{q} 34 ; \mathrm{q} 11),+\operatorname{der}(22) \mathrm{t}(9 ; 22)$.
In March 2006, the patient was enrolled in a study (CAMN107A2101; registered at http://www.clinicaltrials.gov as NCT00109707) (7) and received nilotinib at $800 \mathrm{mg}$ per day. The patient showed a major molecular response of $B C R-A B L$ for 8 years (March 2014) accompanied by several side-effects such as gastrointestinal discomfort and permanent headache.

Loss of major molecular response (BCR-ABL 27\% in peripheral blood) in March 2014 led to a change of treatment protocol replacing nilotinib by dasatinib (100 $\mathrm{mg}$ per day). Due to side-effects such as nausea and vomiting, the treatment was stopped. In August 2014 bone marrow analysis revealed $10 \%$ blasts and $31 \% \quad B C R-A B L$. Therefore, therapeutic intervention was changed to ponatinib (45 mg daily).

Ponatinib treatment ( 6 months) failed to induce a major molecular response (BCR-ABL 19\% bone marrow) and a transition into blast crisis occurred in February 2015 (70\% blasts in peripheral blood). Additional FISH analysis revealed a deletion of chromosome $7[+\operatorname{del}(7)]$. A decrease of mapped reads for chromosome 7 was also revealed by NGS from $11.3 \%$ initially, to $9.1 \%$ in August 2014 and 6.8\% in February 2015.

Therefore, a cytoreductive therapy with cytarabine for 5 days was initiated, followed by one cycle of mitoxantrone, cytarabine and fludarabine (MitoFlaG) due to lack of response. Despite this intense therapy, the blasts were still persistent in the peripheral blood (until March 2014). Therefore a novel induction scheme (azacitidine with bosutinib) (8) was introduced resulting in rapid hematological complete remission (CR).

NGS analyses were performed retrospectively on four samples as well as prospectively on samples of different origins. During the whole time (2004-2015) homozygous (frequency $100 \%$ ) variants in platelet-derived growth factor receptor alpha (PDGFRA), harvey rat sarcoma viral oncogene homolog $(H R A S)$, rearranged during transfection (RET), fibroblast growth factor receptor 3 (FGFR3) genes and heterozygous (frequency 50\%) variants in $A B L 1$ and $R E T$ were revealed. Except for $A B L 1$ (p.K247R), none of the variants lead to an amino acid change (Table I). However, the RET gene (c.2307G >T) and ABL1 (p.K247R) variants are classified as pathogenic according to FATHMM. Despite the fact that $R E T$ (c. $2307 \mathrm{G}>\mathrm{T}$ ) does not lead to an amino acid change, this mutation was frequently found in hematopoietic neoplasms (COSM4418405) (9).

A single nucleotide variant (SNV) affecting the protooncogene MET (p.T1010I) was detected with a frequency of nearly $25 \%$ in the initial samples of 2004. During disease progression, this frequency increased to more than $90 \%$ in March 2014. MET encodes a proto-oncogene and receptor tyrosine kinase located on chromosome 7 . The SNV leads to a nucleotide and amino acid change (p.T1010I), representing a known mutation (COSM707) predicted as pathogenic (FATHMM score 0.98). ClinVar also described this mutation 


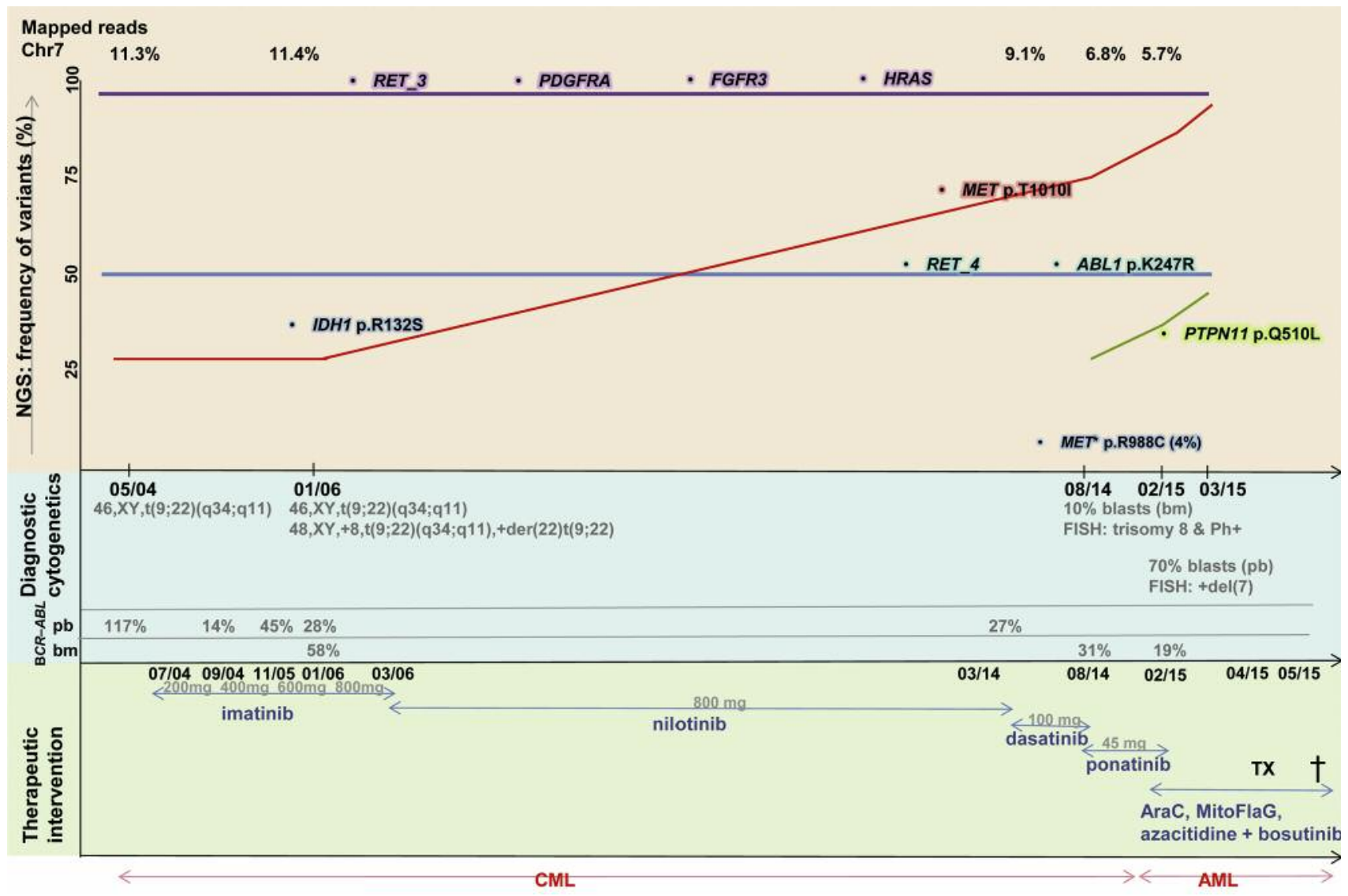

Figure 1. Next-generation sequencing, diagnostic cytogenetics and therapeutic intervention. pb: Peripheral blood; bm: bone marrow; TX: transplantation; Chr: chromosome, AML: acute myeloid leukemia, CML: chronic myeloid leukemia, NGS: next-generation sequencing, FISH: fluorescence in situ hybridization, $t$ : translocation, der: derivative chromosome, AraC: cytarabine, MitoFlaG: mitoxantrone, cytarabine and fludarabine, PDGFRA: platelet derived growth factor receptor alpha, HRAS: harvey rat sarcoma viral oncogene homolog, RET: rearranged during transfection, FGFR3: fibroblast growth factor receptor 3, BCR: breakpoint cluster region, ABL1: tyrosine-protein kinase, MET: proto-oncogene met, PTPN11: tyrosine-protein phosphatase non-receptor type 11, IDH1: isocitrate dehydrogenase 1 .

Table I. Detected gene variants and their allelic frequency, location, single nucleotide variant (SNV) description, coding, amino acid change and predictive score (FATHMM).

\begin{tabular}{|c|c|c|c|c|c|c|c|c|}
\hline Occurrence & Gene & $\begin{array}{l}\text { Allelic } \\
\text { frequency }\end{array}$ & Location & SNV & Coding & $\begin{array}{c}\text { Amino acid } \\
\text { change }\end{array}$ & Allele name & $\begin{array}{l}\text { FATHMM } \\
\text { (score) }\end{array}$ \\
\hline \multirow[t]{6}{*}{ Persisting } & PDGFRA & $100 \%$ & NM_006206.5 & rs 1873778 & c. $1701 \mathrm{~A}>\mathrm{G}$ & - & Novel & N.A. \\
\hline & HRAS & $100 \%$ & NM_001130442.1 & rs12628 & $\mathrm{c} .81 \mathrm{~T}>\mathrm{C}$ & - & COSM249860 & Neutral (0.07) \\
\hline & $R E T$ & $100 \%$ & NM_020975.4 & rs1800861 & c. $2307 \mathrm{G}>\mathrm{T}$ & - & COSM4418405 & Pathogenic (0.79) \\
\hline & $F G F R 3$ & $100 \%$ & NM_001163213.1 & rs7688609 & c. $1959 \mathrm{G}>\mathrm{A}$ & - & Novel & N.A. \\
\hline & $R E T$ & $50 \%$ & NM_020975.4 & rs1800863 & c. $2712 \mathrm{C}>\mathrm{G}$ & - & COSM3751779 & Neutral (0.27) \\
\hline & $A B L 1$ & $50 \%$ & NM_007313.2 & rs 34549764 & c. $740 \mathrm{~A}>\mathrm{G}$ & p.K247R & COSM6005485 & Pathogenic $(0.92)$ \\
\hline \multirow[t]{2}{*}{ Increasing } & MET & $25-90 \%$ & NM_001127500.1 & rs56391007 & c. $3029 \mathrm{C}>\mathrm{T}$ & p.T1010I & COSM707 & Pathogenic (0.98) \\
\hline & PTPN11 & $30-50 \%$ & NM_002834.3 & rs 121918470 & c. $1529 \mathrm{~A}>\mathrm{T}$ & p.Q510L & COSM1318059 & Pathogenic (0.99) \\
\hline \multirow[t]{2}{*}{ Punctual } & $M E T$ & $4 \%$ & NM_001127500.1 & rs34589476 & c. $2962 \mathrm{C}>\mathrm{T}$ & p.R988C & NOCOSMIC988 & N.A. \\
\hline & $I D H 1$ & $43 \%$ & NM_005896.3 & rs121913499 & c. $394 \mathrm{C}>\mathrm{A}$ & p.R132S & COSM28748 & Pathogenic (0.92) \\
\hline
\end{tabular}

- : No amino acid change, N.A.: not available, SNV: single nucleotide variant, FATHMM: Functional Analysis Through Hidden Markov Models, PDGFRA: platelet derived growth factor receptor alpha, HRAS: harvey rat sarcoma viral oncogene homolog, RET: rearranged during transfection, FGFR3: fibroblast growth factor receptor 3, ABL1: tyrosine-protein kinase, MET: proto-oncogene met, PTPN11: tyrosine-protein phosphatase nonreceptor type 11, $I D H 1$ : isocitrate dehydrogenase 1 . 
as likely pathogenic in somatic cells. Furthermore, it was found in tumor samples of lung and thyroid $(10,11)$.

The isocitrate dehydrogenase $1(I D H I)$ gene was found to be affected (frequency 43\%) by a known SNV (COSM28748) predicted as pathogenic (FATHMM score $0.92)$ exclusively in a sample from 2006. IDHI mutations are common in AML (12) and this mutation was also found in patients with AML and multiple myeloma $(13,14)$. A variant in protein tyrosine phosphatase, non-receptor type 11 (PTPN11, frequency 30\%) and a second variant in MET (4\% frequency) were detected in the sample of August 2014. The frequency of the PTPN11 SNV had increased to $50 \%$ by March 2015. This variant results in a nucleotide exchange $(\mathrm{c} .1529 \mathrm{~A}>\mathrm{T})$ and amino acid change (p.Q510L). This missense mutation is known (COSM1318059), classified as pathogenic (FATHMM prediction score 0.99) and was found in AML samples (15). The variant of the $M E T$ gene was at position 116411923 and leads to a nucleotide (c.2962C $>$ T) and amino acid change (p.R988C). ClinVar describes this mutation as likely pathogenic in somatic cells (16).

In March 2015, the patient immediately received full matched allogeneic stem cell transplantation with myeloablative conditioning (busulfane, cyclophosphamide). Unfortunately, the patient developed severe infection complications on day 21 and died on day 28 .

\section{Discussion}

In this article, we present the case of a patient with CML who did not respond to different therapeutic interventions and eventually developed a blast crisis and AML 10 years after diagnosis. Therapeutic protocols had to be changed due to nonresponse or secondary drug resistance and development of $B C R-A B L$-positive cells. NGS analysis revealed silent mutations in the PDGFRA, HRAS, RET and FGFR3 genes and a point-mutation in $A B L 1$ from the initial diagnosis until blast crisis in 2015. $A B L 1$ (p.K247R) was found in samples from patients with CML who failed to achieve response to imatinib (17), and on the other hand, it is described as a polymorphism $(17,18)$. Additionally, we detected an SNV of the MET gene with an initial frequency of nearly $25 \%$, rising to $90 \%$ in March 2015. This transmembrane tyrosine kinase promotes e.g. cell proliferation and survival via phosphatidylinositol 3-kinase $(\mathrm{PI} 3 \mathrm{~K}) /$ protein kinase B (AKT) and mitogen-activated protein kinase (MAPK)/extracellular signal-regulated kinase (ERK) pathway signaling (19). The detected SNV p.T1010I is located on the intracellular juxtamembrane domain and may contribute to persistent pathway activation and tumor pathophysiology (20). This SNV was found in breast (20) and lung cancer (21), Ewing's sarcoma (22) and other cancer types (23). Thus, therapeutic intervention with a MET inhibitor may have had beneficial effects for the patient. However, this mutation is also described as a potential rare polymorphism $(23,24)$.
Despite increasing the dose of imatinib, the patient never achieved CR. During that time, the patient developed more cytogenetic anomalies and an additional clone with +8 and a second Philadelphia chromosome. These chromosomal anomalies are most frequent in CML (25-27). It seems that increased expression of $B C R-A B L$ does play a critical role in promoting genetic instability (3). $B C R-A B L$ regulates DNA damage and response by accumulation of further DNA lesions. As many as $10 \%$ of patients who respond to imatinib finally developed additional karyotype abnormalities, among others, trisomy 8 (28). Additionally, a variant in the $I D H I$ (p.R132S) was detected. Most common variants are heterozygous mutations at p.R132 (29) and this variant was found in a patient with AML (30).

In general, $82 \%$ of patients achieve CR with imatinib, but some patients are non-responders (31). Hence therapy of our patient was changed to second-generation TKI nilotinib. This ABL inhibitor is used after failure of imatinib therapy and induces CR in approximately $50 \%$ of patients (31). The patient also showed complete cytogenetic response, but after 8 years of $C R$ with nilotinib, the frequency of $B C R-A B L$ rose again and the therapy was changed to dasatinib. Dasatinib is an ABL inhibitor which targets more kinases in comparison to imatinib and nilotinib (2). The patient responded to dasatinib, but therapy had to be terminated because of several side-effects. In August 2014, FISH diagnosis revealed the same results as in 2006. It has been demonstrated that treatment with nilotinib or dasatinib leads to improved response rates and reduces the rate of transformation in patients in comparison to imatinib (31). However, NGS analysis detected a SNV in PTPN11, a rise of the previously described $M E T$ mutation, but the $I D H I$ variant was missing. PTPNIl variant is a known driver mutation in AML (32-36). Therapeutic intervention was changed to the third-generation TKI ponatinib. But ultimately the patient developed a blast crisis in February 2015 and therapy was changed to AML protocols. FISH diagnosis revealed monosomy 7 and NGS analysis detected a decrease of mapped reads of chromosome 7 , which was already obvious in the sample of August 2014. Monosomy 7 was reported to correlate with risk of acute leukemia (37).

Apparently, during 10 years of therapeutic intervention, the patient gained additional mutations and clonal selection including genetic shifting occurred. Monosomy 7 , as well as MET (p.T1010L) and PTPN11 (p.Q510L) variants, are especially interesting points. Detection of these findings at an earlier point of time could have increased the chance of recovery of the patient e.g. by use of a MET inhibitor or earlier stem cell transplantation. Thus, this case report shows that the early comprehensive integration of detailed molecular diagnostics can provide significant diagnostic value as well as being helpful for therapeutic protocol decisions. 


\section{References}

1 Rosti G, Castagnetti F, Gugliotta G and Baccarani M: Tyrosine kinase inhibitors in chronic myeloid leukaemia: Which, when, for whom? Nat Rev Clin Oncol 14: 141-154, 2017.

2 Miller GD, Bruno BJ and Lim CS: Resistant mutations in CML and $\mathrm{Ph}+\mathrm{ALL}$ - role of ponatinib. Biol Targets Ther 8: 243-254, 2014.

3 Perrotti D, Jamieson C, Goldman J and Skorski T: Chronic myeloid leukemia: Mechanisms of blastic transformation. J Clin Invest 120: 2254-2264, 2010.

4 Shihab HA, Gough J, Cooper DN, Stenson PD, Barker GLA, Edwards KJ, Day INM and Gaunt TR: Predicting the functional, molecular, and phenotypic consequences of amino acid substitutions using hidden markov models. Hum Mutat 34: 5765, 2013.

5 Kiefer T, Schüler F, Knopp A, Wimmer M, Hirt C, Schaefer HE and Dölken G: A human Burkitt's lymphoma cell line carrying $\mathrm{t}(8 ; 22)$ and $\mathrm{t}(14 ; 18)$ translocations. Ann Hematol 86: 821-830, 2007.

6 McGowan-Jordan J and Simons AMS: ISCN: An International System for Human Cytogenomic Nomenclature. Basel; New York: Karger, 2016.

7 CAMN107A2101. Available from: https://clinicaltrials.gov/ ct2/show/NCT00109707.

8 Ghez D, Micol J-B, Pasquier F, Auger N, Saada V, Spentchian M, Ianotto J-C, Bourhis J-H, Bennaceur-Griscelli A, Terré C, Castaigne S, Rigaudeau S, Rousselot P and de Botton S: Clinical efficacy of second-generation tyrosine kinase inhibitor and 5azacytidine combination in chronic myelogenous leukaemia in myeloid blast crisis. Eur J Cancer 49: 3666-3670, 2013.

9 COSM4418405. Available from: http://cancer.sanger.ac.uk/ cosmic/mutation/overview?id=4418405.

10 COSM707. Available from: http://cancer.sanger.ac.uk/cosmic/ mutation/overview?id=707.

11 NM_001127500.2(MET):c.3029C>T (p.Thr1010Ile). Available from: https://www.ncbi.nlm.nih.gov/clinvar/variation/41624/.

12 Hussaini MO, Mirza A-S, Komrokji R, Lancet J, Padron E and Song J: Genetic landscape of acute myeloid leukemia interrogated by next-generation sequencing: A large cancer center experience. Cancer Genomics Proteomics 15: 121-126, 2018.

13 NM_001282387.1(IDH1):c.394C>A (p.Arg 132Ser). Available from: https://www.ncbi.nlm.nih.gov/clinvar/variation/375893/.

14 COSM28748. Available from: https://cancer.sanger.ac.uk/cosmic/ mutation/overview?id=28748.

15 COSM1318059. Available from: http://cancer.sanger.ac.uk/ cosmic/mutation/overview id $=1318059$.

16 NM_001127500.2(MET):c.2962C>T (p.Arg988Cys). Available from: https://www.ncbi.nlm.nih.gov/clinvar/variation/41623/.

17 Nicolini FE, Chabane K, Cayuela J-M, Rousselot P, Thomas X and Hayette S: The role of the K247R substitution in the ABL tyrosine kinase domain in sensitivity to imatinib. Haematologica 91: 137-138, 2006.

18 Ernst T, Hoffmann J, Erben P, Hanfstein B, Leitner A, Hehlmann R, Hochhaus A and Müller MC: ABL single nucleotide polymorphisms may masquerade as $\mathrm{BCR}-\mathrm{ABL}$ mutations associated with resistance to tyrosine kinase inhibitors in patients with chronic myeloid leukemia. Haematologica 93: 1389-1393, 2008 .
19 Albiges L, Guegan J, Le Formal A, Verkarre V, Rioux-Leclercq N, Sibony M, Bernhard JC, Camparo P, Merabet Z, Molinie V, Allory Y, Orear C, Couvé S, Gad S, Patard JJ and Escudier B: MET Is a potential target across all papillary renal cell carcinomas: Result from a large molecular study of pRCC with CGH array and matching gene expression array. Clin Cancer Res 20: 3411-3421, 2014.

20 Liu S, Meric-Bernstam F, Parinyanitikul N, Wang B, Eterovic AK, Zheng X, Gagea M, Chavez-MacGregor M, Ueno NT, Lei X, Zhou W, Nair L, Tripathy D, Brown PH, Hortobagyi GN, Chen K, Mendelsohn J, Mills GB and Gonzalez-Angulo AM: Functional consequence of the MET-T1010I polymorphism in breast cancer. Oncotarget 6: 2604-2614, 2015.

21 Gautschi O, Stadelmann C, Aebersold-Keller F, König K, Büttner R, Heukamp LC, Betticher D, Baumann C, Buser K, Calderoni A, Casty A, D'addario G, Irlé C, Mamot C, Morant R, Trojan A, Pellicioli E, Jehle-Schwertfeger S, Aebi S and Diebold J: Mutation profiling of lung cancers with long-term response to gefitinib therapy. Oncol Res Treat 38: 560-569, 2015.

22 Jiang Y, Subbiah V, Janku F, Ludwig JA, Naing A, Benjamin RS, Brown RE, Anderson P and Kurzrock R: Novel secondary somatic mutations in Ewing's sarcoma and desmoplastic small round cell tumors. PLoS One 9: e93676, 2014.

23 Zenali M, DeKay J, Liu Z, Hamilton S, Zuo Z, Lu X, Bakkar R, Mills $\mathrm{G}$ and Broaddus R: Retrospective review of MET gene mutations. Oncoscience 2: 533-541, 2015.

24 Pilotto S, Gkountakos A, Carbognin L, Scarpa A, Tortora G and Bria E: MET exon 14 juxtamembrane splicing mutations: Clinical and therapeutical perspectives for cancer therapy. Ann Transl Med 5: 2, 2017.

25 Am C, Coriu D, Arion C, Colita A and Jardan C: The impact of additional cytogenetic abnormalities at diagnosis and during therapy with tyrosine kinase inhibitors in chronic myeloid leukaemia. 8: 502-508, 2015.

26 Loriaux M and Deininger M: Clonal cytogenetic abnormalities in Philadelphia chromosome negative cells in chronic myeloid leukemia patients treated with imatinib. Leuk Lymphoma 45: 2197-2203, 2004.

27 Syed NN, Usman M, Adil S and Khurshid M: Additional chromosomal abnormalities in Philadelphia-positive chronic myeloid leukemia. Hematol Oncol Stem Cell Ther 1: 166-170, 2008.

28 Marktel S, Marin D, Foot N, Szydlo R, Bua M, Karadimitris A, De Melo VAS, Kotzampaltiris P, Dazzi F, Rahemtulla A, Olavarria E, Apperley JF and Goldman JM: Chronic myeloid leukemia in chronic phase responding to imatinib: The occurrence of additional cytogenetic abnormalities predicts disease progression. Haematologica 88: 260-267, 2003.

29 Yang R and Fan Y: IDH1 p. R132 mutations may not be actively involved in the carcinogenesis of hepatocellular carcinoma. Med Sci Monit 20: 247-254, 2014.

30 Yamaguchi S, Iwanaga E, Tokunaga K, Nanri T and Shimomura T: $I D H 1$ and $I D H 2$ mutations confer an adverse effect in patients with acute myeloid leukemia lacking the NPM1 mutation. Eur J Haematol 92: 471-477, 2014.

31 Olshen A, Tang M, Cortes J, Gonen M, Hughes T, Branford S, Quintás-Cardama A and Michor F: Dynamics of chronic myeloid leukemia response to dasatinib, nilotinib, and high-dose imatinib. Haematologica 99: 1701-1709, 2014. 
32 Metzeler KH, Herold T, Rothenberg-thurley M, Amler S, Sauerland MC, Dennis G, Ksienzyk B, Zellmeier E, Hartmann L, Greif PA, Fiegl M, Subklewe M, Bohlander SK, Krug U, Faldum A, Berdel WE, Bernhard W, Thomas B, Hiddemann W and Braess J: Spectrum and prognostic relevance of driver gene mutations in acute myeloid leukemia. Blood 128: 686-699, 2017.

33 Shin S-Y, Lee S-T, Kim H-J, Cho EH, Kim J-W, Park S, Jung $\mathrm{CW}$ and Kim S-H: Mutation profiling of 19 candidate genes in acute myeloid leukemia suggests significance of DNMT3A mutations. Oncotarget 7: 54825-54837, 2016.

34 Loh ML, Reynolds MG, Vattikuti S, Gerbing RB, Alonzo TA, Carlson E, Cheng JW, Lee CM, Lange BJ and Meshinchi S: PTPN11 mutations in pediatric patients with acute myeloid leukemia: Results from the Children's Cancer Group. Leukemia 18: 1831-1834, 2004.

35 Hou HA, Chou WC, Lin LI, Chen CY, Tang JL, Tseng MH, Huang CF, Chiou RJ, Lee FY, Liu MC and Tien HF: Characterization of acute myeloid leukemia with PTPN11 mutation: The mutation is closely associated with NPM1 mutation but inversely related to FLT3/ITD. Leukemia 22: 1075-1078, 2008.
36 Tartaglia M, Niemeyer CM, Song X, Buechner J, Jung A, Hählen $\mathrm{K}$, Hasle H, Licht JD and Gelb BD: Somatic mutations in PTPN11 in juvenile myelomonocytic leukemia, myelodysplastic syndromes and acute myeloid leukemia. Nat Genet 34: 148-151, 2003.

37 Baccarani M, Deininger MW, Rosti G, Hochhaus A, Soverini S, Apperley JF, Cervantes F, Clark RE, Cortes JE, Guilhot F, Hjorth-Hansen H, Hughes TP, Kantarjian HM, Kim DW, Larson RA, Lipton JH, Mahon FX, Martinelli G, Mayer J, Müller MC, Niederwieser D, Pane F, Radich JP, Rousselot P, Saglio G, Saußele S, Schiffer C, Silver R, Simonsson B, Steegmann JL, Goldman JM and Hehlmann R: European LeukemiaNet recommendations for the management of chronic myeloid leukemia: 2013. Blood 122: 872-884, 2013.

Received April 16, 2018

Revised May 28, 2018

Accepted May 30, 2018 